\title{
Expression of miR-132 and miR-212 in prostate cancer and metastatic lymph node: Case report and revision of the literature
}

\author{
Michele Salemi ${ }^{1}$, Angela Pettinato ${ }^{2}$, Filippo Fraggetta ${ }^{2}$, Aldo E. Calogero ${ }^{3}$, Michele Pennisi ${ }^{4}$, \\ Ludovica Pepe ${ }^{4}$, Pietro Pepe ${ }^{4}$ \\ ${ }^{1}$ Oasi Research Institute-IRCCS, Troina (EN), Italy; \\ ${ }^{2}$ Pathology Unit, Cannizzaro Hospital, Catania, Italy; \\ ${ }^{3}$ Section of Endocrinology, Andrology and Internal Medicine, Department of Clinical and Experimental Medicine, University \\ of Catania, Italy; \\ ${ }^{4}$ Urology Unit, Cannizzaro Hospital, Catania, Italy.
}

\begin{abstract}
Summary MicroRNAs (miRNAs) are a class of small, non-coding RNAs that act as key regulators in various physiological and pathological processes as prostate cancer (PCa). In this study we describe molecular evaluation of 132 and 212 miRNAs expression, by Real-time reverse-transcription PCR (qRT-PCR), in a Caucasian man 64-year-old with locally advanced PCa (PSA $160 \mathrm{ng} / \mathrm{ml}$, Gleason score 4+3/ISUP Grade Group 3, clinical stage T3NXM0) who underwent radical retropubic prostatectomy plus extended pelvic lymphadenectomy (LAD) as first step of a multimodal therapeutic treatment. A normal prostate of a 67-year-old man removed by post mortem autopsy was used as a control in the study. The mRNA for this study was conducted on paraffined prostatic sections of: $a$ ) index case of $P C a ; b)$ metastatic lymph node of index case; c) normal prostate. MiRNA-132, miRNA212 and Glyceraldehyde 3-phosphate dehydrogenase (as reference gene) assays were obtained. Definitive specimen showed a pT3bN1R1 stage: acinar cells adenocarcinoma with involvement of the seminal vesicles, multifocal positive surgical margins, Gleason score 8 (4+4/ISUP Grade Group 4), metastases in $5 / 25$ iliac lymph nodes.

An increased expression of miRNA-132 and miRNA-212 in index case of prostatic adenocarcinoma compared to normal prostate tissue was found; moreover, a lower expression of miR-132 and miR-212 in metastatic lymph node compared to primitive PCa and normal prostate tissue was demonstrated. Although a greater number of patients should be evaluated, these data suggest that the biology of the primary PCa, in our clinical case, was different from metastatic lymph node.
\end{abstract}

KEY WORDS: Prostate cancer; miR-132; miR-132; qRT-PCR.

Submitted 2 March 2020; Accepted 15 March 2020

\section{INTRODUCTION}

Prostate cancer (PCa) is the most frequently tumor diagnosed in men; although the screening protocols have increased the early diagnosis for organ-confined PCa, still today, a not negligible number of initial diagnosis is characterized by high risk or metastatic cancer. Patients with high-risk PCa are at an increased risk of PSA failure, need for secondary therapy, metastatic progression and death from $\mathrm{PCa}$; nevertheless, not all high-risk PCa patients have uniformly poor prognosis after definitive therapy (radical prostatectomy or radiotherapy).
Although there is no consensus regarding the optimal treatment of men with high-risk or locally-advanced PCa, in the last years, the multi-modal therapy has demonstrated a clinical advantage for the patients (1) and new clinical trials are ongoing to evaluate the best clinical therapeutic approach for each patient. Therefore, there is the necessity to better understand the prognosis for each high risk $\mathrm{PCa}$ using new parameters (i.e., histological and molecular genetics patterns) $(2,3)$; in this respect, the new techniques of proteomics, RNA and DNA microarrays, have been used to identify and validate accurate diagnostic biomarkers in samples of prostate tissue (4). MicroRNAs (miRNAs) were found aberrantly expressed in PCa (9); MiR-132 has a central role in the processes of cell adhesion, angiogenesis, and tumorigenesis (MIM 610016). Several studies have described a strong association between levels of miR-132 and high Gleason score PCa (10). MiR-212 and mir-132 belong to the same family and are transcribed from a stable gene intron noncoding for proteins (MIM 613487). MiR-212 regulates a sub-set of genes involved in tumor progression in several tumor cell types as PCa (5).

In this study we describe molecular evaluation of 132 and 212 miRNAs expression, by Real-time reverse-transcription PCR (qRT-PCR), in a men with locally-advanced $\mathrm{PCa}$ who underwent radical retropubic prostatectomy (RPP) plus extended pelvic lymphadenectomy (LAD) as first step of a multimodal therapeutic treatment.

\section{Case Report}

A Caucasian 64-year-old man had a total PSA value of $160.0 \mathrm{ng} / \mathrm{ml}$ and positive digital rectal examination. Transperineal extended prostatic biopsies (13) showed in all the cores (12/12) an adenocarcinoma with a Gleason score 7 (4+3/ISUP Grade Group 3) (14); the clinical stadiation performed by total body computed tomography and bone scan showed no presence of metastases (clinical stage pT3NXM0). In January 2019 informed consent was obtained from the patient who accepted to undergo RRP plus extended pelvic LAD as first step of a multimodal therapeutic treatment. Definitive specimen showed a 
Figure 1.

Relative expression value of miR-132 and miR-212: Control 1 and 1 respectively (histogram with double scale); Prostate index case 179.7 and 55.5 respectively; Metastatic lymph node 0.267 and 0.319 respectively.

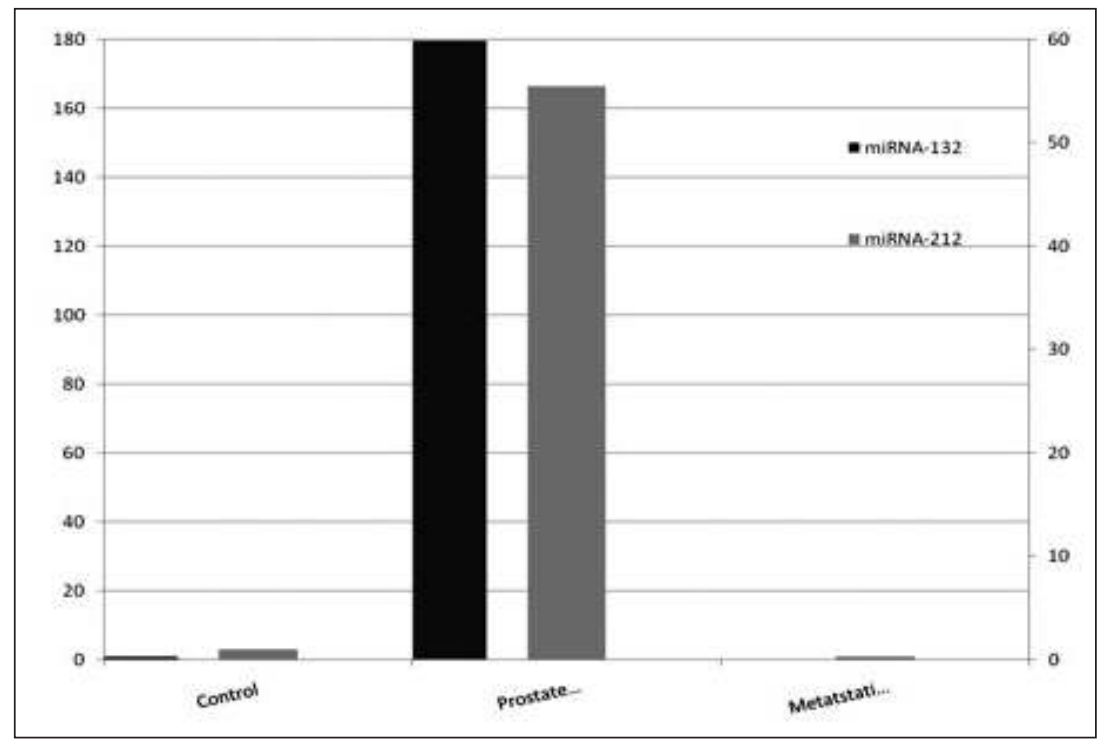

effects by binding to the 3' untranslated region (3'UTR) of their target mRNAs, leading to the inhibition of translation or the degradation of the mRNA, depending on the degree of complementary base pairing.

The miRNAs are deregulated in a wide variety of human cancers; in detail, miR-212 and miR-132 regulate a subset of genes involved in PCa progression and lymph node metastasis.

Data obtained in our case report showed an abnormal over-expression of miR-132 and miR-212 in PCa compared to normal prostatic tissue; also we found a down-expression of miR132 and miR-212 in metastatic lymph node.

Although a greater number of patients should be evaluated, these data suggest that the biology of the primary PCa, in our clinical case, was different from metastatic lymph node.
pT3bN1Rl stage: acinar cells adenocarcinoma with involvement of the seminal vesicles, multifocal positive surgical margins, Gleason score 8 (4+4/ISUP Grade Group 4 ), bilateral metastases in 5/25 iliac lymph nodes. A normal prostate of a 67 -year-old man removed by post mortem autopsy was used as a control in the study. The mRNA for this study was conducted on paraffined prostatic sections of: a) index case of $\mathrm{PCa}$; b) metastatic lymph node of index case; c) and normal prostate. MiR132, miR-212 and Glyceraldehyde 3-phosphate dehydrogenase (GAPDH) (as reference gene) assays were obtained from Applied Biosystems (Carlsbad, CA).

Real-time analysis was performed on Light Cycler 480 (Roche Diagnostics; Mannheim, Germany).

The amplified transcripts were quantified using the comparative CT method and relative quantification analysis data were played using the comparative $\Delta \Delta \mathrm{Ct}$ method included in the Software Version 1.5 supplied with the LightCycler 480.

An increased expression of miRNA-132 and miRNA-212 in index case of prostatic adenocarcinoma compared to normal prostate tissue was found; moreover, a lower expression of miR-132 and 212 in metastatic lymph node compared to primitive PCa and normal prostate tissue (Figure 1) was demonstrated.

Finally, the patient had not clinical complications from RPP; the PSA value was equal to $2.7 \mathrm{ng} / \mathrm{ml} 30$ days from surgery and the patient underwent adjuvant radiotherapy and hormonal therapy (LHRH agonist).

\section{Discussion AND conclusions}

MicroRNAs are short non-coding RNAs involved in several important biological processes through regulation of genes post-transcriptionally; carcinogenesis is one of the key biological processes where miRNAs play important role in the regulation of genes. The miRNAs elicit their

\section{References}

1. Malik ZI, Fenwick JD. Radiotherapy to the primary tumour for patients with metastatic prostate cancer: practice-changing results from STAMPEDE. Clin Oncol. (R Coll Radiol). 2020; pii: S09366555(19)30540-0.

2. D'Antonio A, Caputo A, Fraggetta F, et al. KPNA2/ERG Coexpression is associated with early recurrence in advanced prostate cancers. Appl Immunohistochem Mol Morphol. 2020; 28:62-66.

3. Pepe P, Fraggetta F, Candiano G, Aragona F. Does Ki-67 staining improve quantitative histology in preoperative prostate cancer staging? Arch Ital Urol Androl. 2012; 84:32-35.

4. Hassan O, Ahmad A, Sethi S, Sarkar FH. Recent updates on the role of microRNAs in prostate cancer. J Hematol Oncol. 2012; 5:9.

5. Formosa A, Lena AM, Markert EK, et al. DNA methylation silences miR-132 in prostate cancer. Oncogene 2012; 32:127-134.

\section{Correspondence}

Michele Salemi, MD

michelesalemi@hotmail.it

Oasi Research Institute-IRCCS, Troina (EN) (Italy)

Angela Pettinato, MD

angelapettinato@hotmail.com

Filippo Fraggetta, MD

Pathology Unit, Cannizzaro Hospital, Catania (Italy)

filippofra@hormail.com

Aldo E. Calogero, MD

acalogero@hotmail.com

Section of Endocrinology, Andrology and Internal Medicine,

Department of Clinical and Experimental Medicine,

University of Catania, Catania (Italy)

Michele Pennisi, MD

michelepennisi2@virgilio.it

Ludovica Pepe, MD

ludovicapepe@hotmail.com

Pietro Pepe, MD (Corresponding Author)

piepepe@hotmail.com

Urology Unit, Cannizzaro Hospital

Via Messina 829, Catania (Italy) 